TITLE:

\title{
Acceleration statistics as measures of statistical persistence of streamlines in isotropic turbulence
}

$\operatorname{AUTHOR}(\mathrm{S}):$

Goto, S; Osborne, DR; Vassilicos, JC; Haigh, JD

\section{CITATION:}

Goto, S ... [et al]. Acceleration statistics as measures of statistical persistence of streamlines in isotropic turbulence. Physical Review E 2005, 71(1): 015301.

ISSUE DATE:

2005-01

URL:

http://hdl.handle.net/2433/39910

RIGHT:

Copyright 2005 American Physical Society 
PHYSICAL REVIEW E 71, 015301(R) (2005)

\title{
Acceleration statistics as measures of statistical persistence of streamlines in isotropic turbulence
}

\author{
S. Goto, ${ }^{1, *}$ D. R. Osborne, ${ }^{1,2}$ J. C. Vassilicos, ${ }^{1}$ and J. D. Haigh ${ }^{2}$ \\ ${ }^{1}$ Turbulence and Mixing Group, Department of Aeronautics, Imperial College, London, SW7 2AZ, United Kingdom \\ ${ }^{2}$ Space and Atmospheric Physics, Department of Physics, Imperial College, London, SW7 2AZ, United Kingdom
}

(Received 29 July 2004; published 12 January 2005)

\begin{abstract}
We introduce the velocity $\boldsymbol{V}_{s}$ of stagnation points as a means to characterize and measure statistical persistence of streamlines. Using theoretical arguments, direct numerical simulations (DNS), and kinematic simulations (KS) of three-dimensional isotropic turbulence for different ratios of inner to outer length scales $L / \eta$ of the self-similar range, we show that a frame exists where the average $\left\langle\boldsymbol{V}_{s}\right\rangle=\mathbf{0}$, that the rms values of acceleration, turbulent fluid velocity, and $V_{s}$ are related by $L a^{\prime} / u^{\prime 2} \sim\left(V_{s}^{\prime} / u^{\prime}\right)(L / \eta)^{2 / 3+q}$, and that $V_{s}^{\prime} / u^{\prime} \sim(L / \eta)^{q}$ with $q=-1 / 3$ in Kolmogorov turbulence, $q=-1 / 6$ in current DNS, and $q=0$ in our KS. The statistical persistence hypothesis is closely related to the Tennekes sweeping hypothesis.
\end{abstract}

DOI: 10.1103/PhysRevE.71.015301

PACS number(s): 47.27.Ak, 47.27.Qb, 47.27.Gs, 92.10.Lq

Accelerations are central to fluid flow. Acceleration statistics are central to turbulence dynamics, turbulent mixing, and transport, and are also important where the turbulence controls droplet growth, chemical reactions, combustion, and other processes (e.g., [1,2]). A key statistic is the acceleration variance. Its value and scaling with Reynolds number are essential to stochastic Lagrangian models and to Lagrangian probability density-function models of turbulent diffusion if these models are to incorporate finite Reynolds number effects [2]. In this paper we argue that the Reynolds number scaling of the turbulence acceleration statistics, in particular the acceleration variance, are also important as measures of the statistical persistence of streamlines.

The persistence of streamlines is central to turbulent pair diffusion [3-6]. It may also be important for one-particle two-time Lagrangian turbulent statistics [7]. If streamlines are persistent enough in time, fluid element trajectories approximately follow them for significantly long times. Hence, initially close fluid element pairs typically separate when they encounter a region of highly curved diverging streamlines around a straining stagnation point. However, streamlines and their persistence are not Galilean invariant. This issue motivated [6] to suggest that the dependence of turbulent diffusion on streamline structure fully emerges only in the frame of reference where streamline persistence is maximized in some statistical sense. The assumption that such a frame exists, and that the statistical persistence of the streamline topology in this frame is long enough to leave its defining imprint on turbulent diffusion, is termed "statistical persistence hypothesis [6]." This is a powerful hypothesis as it leads to predictions supported by DNS and KS such as $\gamma$ $=2 d / D_{s}$ (see $\left.[5,6]\right)$, which relate the Richardson pairdiffusion exponent $\gamma$ (a Lagrangian quantity) in $d$-dimensional turbulence to the fractal dimension $D_{s}$ of the spatial distribution of stagnation points (an Eulerian quantity) in the privileged frame of the statistical persistence hypothesis. For isotropic turbulence, [6] argued that this frame

*Permanent address: Department of Mechanical Engineering, Kyoto University, Kyoto 606-8501, Japan. is the one where the mean fluid velocity vanishes. The purpose of this paper is to mathematically formulate the statistical persistence of streamlines as well as the statistical persistence hypothesis (statistical persistence of streamlines maximized in a well-chosen frame) and to argue that, in isotropic turbulence, they are both increasingly valid for increasing Reynolds number.

It is the persistence of highly curved diverging streamlines around straining stagnation points that is important for turbulent diffusion, and it is therefore critical to consider the speed with which stagnation points move in space. Given an arbitrary frame of reference, the fluid velocity $\boldsymbol{u}$ at a stagnation point $\boldsymbol{s}(t)$ at time $t$ vanishes, i.e., $\boldsymbol{u}(\boldsymbol{s}, t)=\mathbf{0}$, and remains so for as long as this stagnation point exists. Hence, during the stagnation point's lifetime, $\quad \mathbf{0}=d / d t \boldsymbol{u}(\boldsymbol{s}, t)=\partial \boldsymbol{u} / \partial t$ $+\boldsymbol{V}_{s} \cdot \nabla \boldsymbol{u}$ at position $\boldsymbol{s}$ and time $t$, and $\boldsymbol{V}_{s} \equiv d \boldsymbol{s} / d t$ is the stagnation point velocity. The fluid acceleration is defined as $\boldsymbol{a}$ $\equiv \partial \boldsymbol{u} / \partial t+\boldsymbol{u} \cdot \nabla \boldsymbol{u}$ at all positions $\boldsymbol{x}$ and times $t$. Setting $\boldsymbol{x}=\boldsymbol{s}$, we obtain

$$
\boldsymbol{a}=-\boldsymbol{V}_{s} \cdot \nabla \boldsymbol{u}
$$

at any time $t$ and any stagnation point $s(t)$ of the flow. Using Cramer's rule, and assuming that $\operatorname{det}(\partial \boldsymbol{u} / \partial x, \partial \boldsymbol{u} / \partial y, \partial \boldsymbol{u} / \partial z)$ $\neq 0$, this equality can be inverted,

$$
\boldsymbol{V}_{s}=-\frac{\left[\operatorname{det}\left(\boldsymbol{a}, \frac{\partial \boldsymbol{u}}{\partial y}, \frac{\partial \boldsymbol{u}}{\partial z}\right), \operatorname{det}\left(\frac{\partial \boldsymbol{u}}{\partial x}, \boldsymbol{a}, \frac{\partial \boldsymbol{u}}{\partial z}\right), \operatorname{det}\left(\frac{\partial \boldsymbol{u}}{\partial x}, \frac{\partial \boldsymbol{u}}{\partial y}, \boldsymbol{a}\right)\right]}{\operatorname{det}\left(\frac{\partial \boldsymbol{u}}{\partial x}, \frac{\partial \boldsymbol{u}}{\partial y}, \frac{\partial \boldsymbol{u}}{\partial z}\right)} .
$$

Note that relations (1) and (2) hold in any frame of reference. What changes with frame is the number and positions of stagnation points (where these relations hold).

These relations are kinematic and are the fundamental link we use here between accelerations and the persistence of stagnation points measured by $\boldsymbol{V}_{s}$. We formulate the concept of statistical persistence of streamlines on the basis of (1). Assuming, as is reasonable in fully developed isotropic turbulence [8], that the kinetic-energy dissipation rate per unit 
mass, $\epsilon$, scales as $u^{\prime 3} / L$ (where $u^{\prime}$ is the turbulence velocity fluctuations rms, and $L$ is an outer length scale of the turbulence) and that the velocity gradients in (1) scale with the inner length scale $\eta$ and the small-scale velocity $u_{\eta}$ $\sim(\epsilon \eta)^{1 / 3}$, then the acceleration rms $a^{\prime}$ is related to the rms $V_{s}^{\prime}$ of $\boldsymbol{V}_{s}$ by

$$
V_{s}^{\prime} \sim a^{\prime} \tau_{\eta},
$$

where $\tau_{\eta} \sim \eta / u_{\eta}$, or equivalently

$$
L a^{\prime} / u^{\prime 2} \sim\left(V_{s}^{\prime} / u^{\prime}\right)(L / \eta)^{2 / 3} .
$$

Strictly, $a^{\prime}$ is the acceleration rms over all stagnation points, but it is also equal to the acceleration rms over the entire field because Galilean transformations leave $a^{\prime}$ unchanged even though they cause the rms statistics to be calculated over different ensembles of points. Our DNS and KS calculations of $a^{\prime}$ and $V_{s}^{\prime}$ support this view. The Kolmogorov scaling of $a^{\prime}$ (where $a^{\prime}$ is determined by $\epsilon$ and $\eta$ ) is $L a^{\prime} / u^{\prime 2}$ $\sim(L / \eta)^{1 / 3}$ and has been corroborated [using $\eta \sim\left(\nu^{3} / \epsilon\right)^{1 / 4}$ where $\nu$ is the kinematic viscosity] by laboratory measurements of acceleration statistics in isotropic turbulence [9] in the range $900<\operatorname{Re}_{\lambda}<2000$, where $\operatorname{Re}_{\lambda}$ is the Taylor-lengthbased Reynolds number. This scaling implies $V_{s}^{\prime} / u^{\prime}$ $\sim(L / \eta)^{-1 / 3}$, which means that the movement of stagnation points (characterized by $V_{s}^{\prime}$ ) is, statistically, increasingly slower than that of fluid elements (characterized by $u^{\prime}$ ) as the Reynolds number increases. In this statistical sense, streamline curvature around stagnation points becomes increasingly persistent and fluid element trajectories can follow the curvature of these streamlines for an increasingly significant time. This interpretation assumes, as we confirm in our DNS and KS studies of isotropic turbulence which we report below, that in the frame where the mean flow vanishes, the average of $\boldsymbol{V}_{s}$ over the entire flow is 0, i.e., $\left\langle\boldsymbol{V}_{s}\right\rangle=\mathbf{0}$. In other inertial frames, $\left\langle\boldsymbol{V}_{s}\right\rangle$ is not zero but proportional to the velocity $\boldsymbol{U}$ of the frame relative to the one where the mean flow vanishes, thus reducing the persistence of streamlines in such other frames. (Stagnation points in these frames $F$ correspond to points with fluid velocity $\boldsymbol{U}$ in the frame $F_{0}$ where $\left\langle\boldsymbol{V}_{s}\right\rangle=\mathbf{0}$. The average acceleration over these points in frame $F_{0}$ is $\langle\boldsymbol{a} \mid \boldsymbol{U}\rangle$, i.e., the average of $\boldsymbol{a}$ conditional on points where the fluid velocity is $\boldsymbol{U}$, and is equal to the average acceleration over the corresponding stagnation points in frame $F$ which follows from (1) and is given by $-\left\langle\boldsymbol{V}_{s} \cdot \nabla \boldsymbol{u}\right\rangle$. The DNS study which we report below indicates that $\langle\boldsymbol{a} \mid \boldsymbol{U}\rangle \propto \boldsymbol{U}$ for all Reynolds numbers tested, thus suggesting $\left\langle\boldsymbol{V}_{s}\right\rangle \propto \boldsymbol{U}$, a proportionality relation confirmed by our DNS with a negative proportionality coefficient.) In conclusion, a frame exists where the statistical persistence of streamlines is maximized and where the statistical persistence hypothesis is valid provided that $V_{s}^{\prime}$ is much smaller than $u^{\prime}$; this frame is the one where the average of $\boldsymbol{V}_{s}$ is zero, hence the one where the mean flow vanishes as argued by [6].

DNS studies of three-dimensional isotropic turbulence support the non-Kolmogorov scaling $L a^{\prime} / u^{\prime 2} \sim(L / \eta)^{1 / 2}$ in the range $40<\operatorname{Re}_{\lambda}<230$ (see [10] and note that $\epsilon \sim u^{\prime 3} / L$ is approximately valid in such simulations with such Reynolds numbers [8] where, also, $\left.\eta \sim\left(\nu^{3} / \epsilon\right)^{1 / 4}\right)$ and therefore imply
$V_{s}^{\prime} / u^{\prime} \sim(L / \eta)^{-1 / 6}$ in that range. This also means that stagnation points become increasingly persistent as Reynolds number increases, but at a rate slower than that of Kolmogorov turbulence. Defining an exponent, $q$, by

$$
L a^{\prime} / u^{\prime 2} \sim(L / \eta)^{2 / 3+q} \sim \operatorname{Re}_{\lambda}^{1+3 q / 2}
$$

[where $\operatorname{Re}_{\lambda} \sim(L / \eta)^{2 / 3}$ has been used], it follows from (4) that (5) is equivalent to

$$
V_{s}^{\prime} / u^{\prime} \sim(L / \eta)^{q} \sim \operatorname{Re}_{\lambda}^{3 q / 2} ;
$$

$q>0$ corresponds to absence and $q<0$ corresponds to presence of statistical persistence of streamlines (in the sense that frames exist where streamlines are statistically fairly persistent and a privileged frame also exists (the one where $\left\langle\boldsymbol{V}_{s}\right\rangle$ $=\mathbf{0})$ where this statistical persistence is maximized and the statistical persistence hypothesis holds). In the remainder of this paper we use DNS and KS to confirm and further explore the behavior of $\boldsymbol{V}_{s}$, the Reynolds number scalings of $L a^{\prime} / u^{\prime 2}$ and $V_{s}^{\prime} / u^{\prime}$, and the relation between these scalings via (4). An advantage of formula (2) is that it can be used to calculate $\boldsymbol{V}_{s}$ from Eulerian snapshots of DNS and KS velocity fields without having to track the motion of stagnation points.

We use DNS data of nondecaying homogeneous isotropic three-dimensional incompressible turbulence generated by a standard spectral code (the magnitudes of a few low wavenumber modes are kept constant) with grid resolution of about $2 \eta$ (except for the highest Reynolds number where it is about $3 \eta$ ) and compute stagnation points and their velocities $\boldsymbol{V}_{s}$ in instantaneous velocity fields for $\mathrm{Re}_{\lambda}$ ranging from 57 to 250 (see [11] for the DNS numerical scheme and parameters). We use the Newton-Raphson method to find all stagnation points. This is an iterative method and requires starting points, which have been taken over the DNS field's entire domain at points separated by the numerical grid width. For every Reynolds number, we calculate $\left\langle\boldsymbol{V}_{s}\right\rangle$ in various frames $F,\langle\boldsymbol{a} \mid \boldsymbol{u}\rangle$ in frame $F_{0}$, and also $a^{\prime}, V_{s}^{\prime}$, the integral length scale $L$ and $\eta$ (we calculate $-\nabla p+\nu \nabla^{2} \boldsymbol{u}$ to obtain $\boldsymbol{a}$. If we wrongly approximate $\boldsymbol{a}=-\boldsymbol{\nabla} p$, then we find $\langle\boldsymbol{a} \mid \boldsymbol{u}\rangle=0$ ). Our results show for all our Reynolds numbers, that $\langle\boldsymbol{a} \mid \boldsymbol{u}\rangle$ $\approx-u^{\prime} / 2 L(\boldsymbol{u}-\langle\boldsymbol{u}\rangle)$ [see Fig. 1(a); note that we observe components $\left\langle a_{i} \mid u_{j}\right\rangle$ to be 0 only if $\left.i \neq j\right]$ and that $\left\langle\boldsymbol{V}_{s}\right\rangle=\mathbf{0}$ only when $\boldsymbol{U}=\mathbf{0}$ [see Fig. 1(b)]. We also plot $L a^{\prime} / u^{\prime 2}$ and $V_{s}^{\prime} /\left(a^{\prime} \tau_{\eta}\right)$ as functions of $L / \eta$ [see Fig. 1(c)]. In agreement with previous DNS studies (see [10] and references therein) we obtain $L a^{\prime} / u^{\prime 2} \sim(L / \eta)^{1 / 2}$ (i.e., $q=-1 / 6$ ) and confirm (3) over our DNS range of Reynolds numbers. By the way, from $\langle\boldsymbol{a} \mid \boldsymbol{U}\rangle=-\left\langle\boldsymbol{V}_{s} \cdot \nabla \boldsymbol{u}\right\rangle$ and $\langle\boldsymbol{a} \mid \boldsymbol{U}\rangle \approx-\left(u^{\prime} / 2 L\right) \boldsymbol{U}$ one might expect $\left\langle\boldsymbol{V}_{s}\right\rangle \propto \boldsymbol{U}$ with a constant of proportionality of decreasing absolute value for increasing $\operatorname{Re}_{\lambda}$, as we indeed observe. What is unexpected (but is left for future investigation) is the rate of this decrease; Figure 1(b) suggests $\left\langle\boldsymbol{V}_{s}\right\rangle=-b(L / \eta)^{-1 / 3} \boldsymbol{U}$, where $b$ is a positive dimensionless universal constant. At any rate, our DNS suggest that $\left\langle\boldsymbol{V}_{s}\right\rangle / u^{\prime} \rightarrow \mathbf{0}$ and $V_{s}^{\prime} / u^{\prime} \rightarrow 0$ as $\operatorname{Re}_{\lambda} \rightarrow \infty$. From (1), this implies that as $\operatorname{Re}_{\lambda} \rightarrow \infty$, stagnation points tend to become nonmoving zero-acceleration points. 

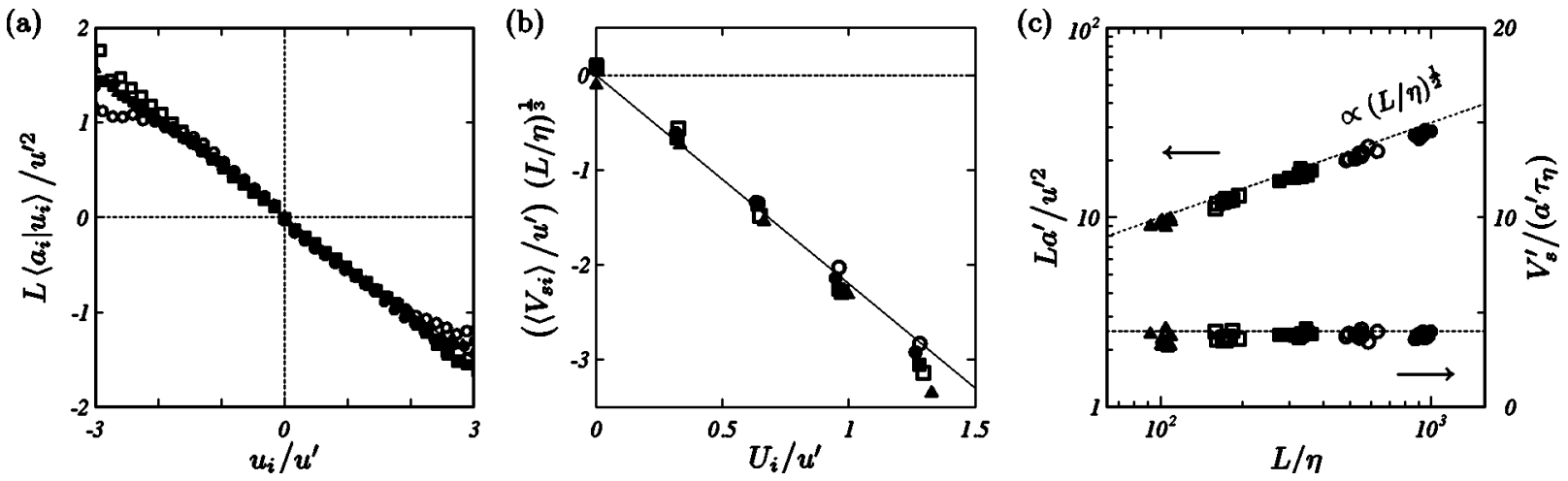

FIG. 1. Statistics of acceleration and stagnation point velocity (DNS). Solid triangles, $\operatorname{Re}_{\lambda}=57$; open squares, 83; solid squares, 120; open circles, 180; solid circles, 250. (a) Conditional average of acceleration in the frame $F_{0}$. (b) Average stagnation point velocity in the moving frame with $\boldsymbol{U}$ relative to $F_{0}$. (c) Rms acceleration and rms stagnation velocity; here we plot results of ten different snapshots from each simulation. $L / \eta$ fluctuates in time, which explains the small scatter around its average.

To gain further insight into $\left\langle\boldsymbol{V}_{s}\right\rangle$ and the validity of relations (4)-(6) and how they depend on specific properties of the underlying flow, we now study synthetic velocity fields, namely, KS, where the spectrum and the time-dependence can be modified at will $[3-5,12]$. Such a study cannot be carried out with DNS where the spectrum and timedependence of the flow are determined by the Navier-Stokes dynamics and cannot be tampered with. An additional advantage of KS is that the Lagrangian statistics it produces compare well with various DNS and laboratory results (see [12] and references therein). Finally, because of the dramatic decimation in number of modes, it is possible with $\mathrm{KS}$ to explore scalings with $L / \eta$ up to extremely large $L / \eta$ values (here $10^{6}$ ) which are out of reach of current DNS.

In our KS we use three-dimensional turbulentlike velocity fields of the form (see [12] for fuller details)

$$
\boldsymbol{u}=\sum_{n=1}^{N_{k}} \boldsymbol{A}_{n} \cos \left(\boldsymbol{k}_{n} \cdot \boldsymbol{x}+\omega_{n} t\right)+\boldsymbol{B}_{n} \sin \left(\boldsymbol{k}_{n} \cdot \boldsymbol{x}+\omega_{n} t\right),
$$

where $N_{k}$ is the number of modes, and the directions and orientations of $\boldsymbol{A}_{n}$ and $\boldsymbol{B}_{n}$ are chosen randomly and uncorrelated with the directions and orientations of all other wave number modes but perpendicular to $\boldsymbol{k}_{n}$. The distribution of wave numbers is geometric, specifically $k_{n} \equiv\left|\boldsymbol{k}_{n}\right|=k_{1} 1.07^{n-1}$. The velocity field is incompressible by construction, and also statistically stationary, homogeneous, and isotropic as shown by $[3,4]$. The amplitudes of the vectors $\boldsymbol{A}_{n}$ and $\boldsymbol{B}_{n}$ are determined from the energy spectrum $E\left(k_{n}\right)$ prescribed to be of the form

$$
E(k)=\frac{3(p-1) u^{\prime 2}}{2(L / 2 \pi)^{p-1}} k^{-p}
$$

in the range $2 \pi / L=k_{1} \leqslant k \leqslant k_{N_{k}}=2 \pi / \eta$, and $E(k)=0$, otherwise. The ratio $L / \eta$ is increased by increasing $N_{k}$. Following $[4,12]$, we set $\omega_{n}=\lambda u^{\prime} k_{n}$, for different values of the dimensionless parameter $\lambda$.

From (1) we can derive a generalized form of (4) within the framework of KS by assuming that the velocity gradients in (1) scale with the inner length scale $\eta$ and the small-scale velocity $u_{\eta} \sim u^{\prime}(\eta / L)^{p-1 / 2}$. This leads to $L a^{\prime} / u^{\prime 2} \sim\left(V_{s}^{\prime} / u^{\prime}\right)$ $\times(L / \eta)^{3-p / 2}$ which generalizes (4) and to the statement that $L a^{\prime} / u^{\prime 2} \sim(L / \eta)^{3-p / 2+q}$ is equivalent to $V_{s}^{\prime} / u^{\prime} \sim(L / \eta)^{q}$ which generalizes (5) and (6).

KS runs with $p=5 / 3$, values of $L / \eta$ ranging between 10 and $10^{3}$, and $\lambda=0,0.5,5$, all lead to $\langle\boldsymbol{a} \mid \boldsymbol{u}\rangle=\mathbf{0}$ and to $\left\langle\boldsymbol{V}_{s}\right\rangle$ $=\mathbf{0}$ in frame $F_{0}$. In frames $F,\left\langle\boldsymbol{V}_{s}\right\rangle=-\boldsymbol{U}$. In KS, $\boldsymbol{V}_{s}$ is uncorrelated with $\nabla \boldsymbol{u}$ so that $\left\langle\boldsymbol{V}_{s} \cdot \nabla \boldsymbol{u}\right\rangle=\left\langle\boldsymbol{V}_{s}\right\rangle \cdot\langle\nabla \boldsymbol{u}\rangle=0$ in agreement with $\mathbf{0}=\langle\boldsymbol{a} \mid \boldsymbol{U}\rangle=-\left\langle\boldsymbol{V}_{s} \cdot \boldsymbol{\nabla} \boldsymbol{u}\right\rangle$. Runs with $p=1.4,5 / 3,1.8$, values of $L / \eta$ ranging between 10 and $10^{6}$, and $\lambda=0,0.5,5$ also lead to $L a^{\prime} / u^{\prime 2} \sim(L / \eta)^{3-p / 2}$ [see Fig. 2(a)] and therefore $q$ $=0$, and to $V_{s}^{\prime} / u^{\prime}=c$ where $c$ is a dimensionless constant independent of $L / \eta$, which confirms that $q=0$ [see Fig. 2(b)]. (In all our KS cases, $5 \times 10^{6}$ starting points for the NewtonRaphson method are chosen over the same volume $L_{s}^{3}$, where $L_{s}$ is the $L$ corresponding to the largest $L / \eta$ tried, keeping $\eta$ constant.)

The result $\langle\boldsymbol{a} \mid \boldsymbol{u}\rangle=\mathbf{0}$ reflects the lack of dynamics and related lack of correlations between Fourier modes in KS. Turbulence dynamics seem to generate restoring accelerations which are anticorrelated with velocities [see Fig. 1(a)].

The constant $c$ turns out to be an increasing function of $\lambda$ (from our simulations, $c \propto \lambda$ ) and, as expected, $c=0$ for $\lambda$ $=0$. Hence, in our KS isotropic turbulence where $q=0$, the statistical persistence of streamlines is measured by $c$ and is a direct reflection of the unsteadiness parameter $\lambda$. In spite of $q$ being different from the Kolmogorov value $-1 / 3$, Richardson exponents $\gamma=2 d / D_{s}$ (in particular $\gamma=3$ for $p=5 / 3$ ) are observed in KS but only for small values of $\lambda[4,5]$ thus confirming the view that these exponents require some statistical persistence of streamlines to be realized [6].

Finally, it is worth recalling the Tennekes sweeping hypothesis [13] which states that the dissipative eddies are swept past an Eulerian observer in a time much shorter than the time scale characterizing their own dynamics. The statistical persistence hypothesis, the validity of which we confirm in this paper, states that there exists a frame where $\left\langle\boldsymbol{V}_{s}\right\rangle=\mathbf{0}$ and $V_{s}^{\prime} \ll u^{\prime}$; from (3), it therefore follows that $a^{\prime} \ll u^{\prime} / \tau_{\eta}$. In this sense, the accelerations are small, which is a way to restate the Tennekes sweeping hypothesis. Indeed, the time 
GOTO et al.

PHYSICAL REVIEW E 71, 015301(R) (2005)

(a)

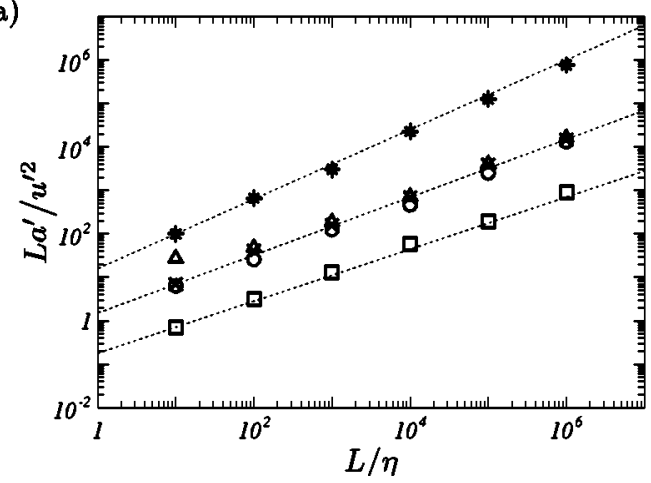

(b)

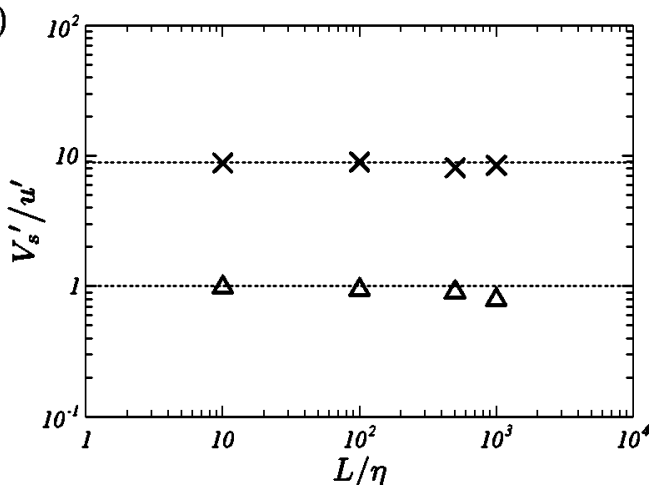

FIG. 2. Statistics of acceleration and stagnation point velocity (KS). (a) Rms acceleration scaling with $L / \eta$. For $p=5 / 3$; crosses, $\lambda=0$; circles, 0.5; triangles, 5.0. For $\lambda=0.5$; asterisks, $p=1.4$; squares, 1.8. (Data sets for these $p$ are shifted with respect to the vertical axis by factor 10 for $p=1.4$ and by 0.1 for 1.8 .) The slopes of the lines are $(3-p) / 2$. (b) Variation of rms stagnation point velocity with $L / \eta$. Triangles, $\lambda=0.5$; crosses, $\lambda=5.0$.

needed for dissipative eddies to sweep past an Eulerian observer is $\eta / u^{\prime}$, which is therefore much smaller than $u_{\eta} / a^{\prime}$, the time which characterizes the dynamics of these eddies. This is the Tennekes sweeping hypothesis derived from the statistical persistence of streamlines. Alternatively, stagnation points mark regions of the flow where there is no sweeping. According to [13], statistics which are taken so as to remove the sweeping effect depend only on the small-scale dynamics, and this must therefore be the case of $V_{s}^{\prime}$. In Kolmogorov turbulence, the scaling of these small-scale dynamics is determined by $\epsilon$ and $\nu$ which therefore implies $V_{s}^{\prime}$ $\sim(\epsilon \nu)^{1 / 4}$, in agreement with $q=-1 / 3$ and the statistical persistence hypothesis. In the present KS, however, sweeping of smaller-scale turbulence by larger-scale eddies is absent, and stagnation points correspond to regions where zeros of Fourier modes congregate. These Fourier modes move together with velocity $\omega(k) / k$. Hence, stagnation points typically move with that same speed and $V_{s}^{\prime} \sim \omega(k) / k \sim \lambda u^{\prime}$ in agreement with our KS results $q=0$ and $c \propto \lambda$.

The statistical persistence of streamlines seems to be a reformulation and generalization of the Tennekes sweeping hypothesis in terms of streamline topology and its persistence. Also, in conjunction with the kinematic relation (1), Kolmogorov dimensional analysis implies the existence of a coherent flow structure, namely, the persistent multiple-scale stagnation point structure of the turbulence. Elsewhere [14] we argue that the mean lifetime of stagnation points is of the order of the integral time scale of the flow.

We acknowledge support from the Japanese Ministry of Education, Culture, Sports, Science and Technology; NERC; Grant No. RGC/KHUST60120 and the Royal Society of London.
[1] R. A. Shaw, Annu. Rev. Fluid Mech. 35, 183 (2003).

[2] S. B. Pope, Annu. Rev. Fluid Mech. 26, 23 (1994).

[3] J. C. H. Fung, J. C. R. Hunt, N. A. Malik, R. J. Perkins, J. Fluid Mech. 236, 281 (1992).

[4] J. C. H. Fung and J. C. Vassilicos, Phys. Rev. E 57, 1677 (1998).

[5] J. Dávila and J. C. Vassilicos, Phys. Rev. Lett. 91, 144501 (2003).

[6] S. Goto and J. C. Vassilicos, New J. Phys. 6, 65 (2004)

[7] M. A. I. Khan and J. C. Vassilicos, Phys. Fluids 16, 216 (2004).
[8] B. Pearson, T. A. Youse, N. E. L. Haugen, A. Bradenburg, and P.-A. Krogstad (unpublished).

[9] A. La Porta, G. A. Voth, A. M. Crawford, J. Alexander, and E. Bodenschatz, Nature (London) 409, 1017 (2001).

[10] P. Vedula and P. K. Yeung, Phys. Fluids 11, 1208 (1999).

[11] S. Goto and S. Kida, Fluid Dyn. Res. 33, 403 (2003).

[12] D. R. Osborne, J. C. Vassilicos, and J. Haigh, Phys. Fluids (to be published).

[13] H. Tennekes, J. Fluid Mech. 67, 561 (1975).

[14] J. C. Vassilicos et al., Proceedings of the IUTAM Symposium, Kyoto, Japan, 2004 (unpublished). 